\title{
An Accelerating Universe with No Dark Energy
}

\author{
Daniel E. Friedmann \\ 2578 West 7th Avenue, Vancouver, Canada \\ Email: friedmann.daniel@alumni.ubc.ca
}

How to cite this paper: Friedmann, D.E. (2018) An Accelerating Universe with No Dark Energy. Open Access Library Journal, 5: e4740.

https://doi.org/10.4236/oalib.1104740

Received: June 26, 2018

Accepted: August 17, 2018

Published: August 20, 2018

Copyright (c) 2018 by author and Open Access Library Inc.

This work is licensed under the Creative Commons Attribution International License (CC BY 4.0).

http://creativecommons.org/licenses/by/4.0/

(c) (†) Open Access

\begin{abstract}
The original application of general relativity to the universe showed that the universe is expanding, albeit at a decreasing rate. Supernova data have established that although early in its history the universe was expanding at a decreasing rate, the rate of expansion has been increasing for the past several billion years. Einstein's equations were modified by adding the cosmological constant to make the expansion of the universe accelerate and fit the data, giving birth to the notion of dark energy. However, there is to date no good explanation of dark energy. This paper proposes that Einstein's original equations be left alone and that instead, the model assumed for the expanding universe be changed: from a single uniformly isotropic expanding space universe of constant mass to a similarly expanding universe surrounded by an isotropic, uniform shell. The overall mass of the structure remains constant. This new geometry produces the observed expansion behavior of the universe and is simply a result of different initial conditions.
\end{abstract}

\section{Subject Areas}

Theoretical Physics

\section{Keywords}

Cosmology: Distance Scale, Dark Energy, Theory, Cosmological Parameters, Large-Scale Structure of Universe

\section{Introduction}

It has been observed that the universe is expanding, for half of its history at a decelerating rate (after a very early period of inflation), but for the past six billion years or so, at an accelerating rate, see for example, [1] and [2]. The determination that expansion is occurring is a direct result of solving Einstein's equations [3]. However, the solution to these equations shows that expansion decelerates through the full history of the universe. Thus, without some modification, 
there is no way to fit the observations from supernova data that demand a new view: a universe expanding at an increasing rate during the past several billion years [2].

The current approach to resolving the lack of congruence between general relativity and observation has been to add a cosmological constant to the equations, creating a repulsive force. The resulting concordance $\Lambda \mathrm{CDM}$ (Lambda Cold Dark Matter) model [4] posits an unknown form of energy with negative pressure and an energy density 123 orders of magnitude different from theoretical expectations. The $\Lambda \mathrm{CDM}$ paradigm reproduces most observations, although so far no plausible candidate for dark energy has emerged and some issues remain [5]. In particular, recent local measurements of the Hubble constant [6] are up to $3.4 \sigma$ higher than the value derived from Planck's observations [4] of the cosmic microwave background.

In addition to positing dark energy, some have tried to account for the differences by modifying gravitational theory (for a review, see [7]). However, Einstein's theoretical predictions continue to be proven right.

Another approach has been based on the fact that local inhomogeneities influence the overall expansion rate and can produce acceleration (for a review, see [8] and [9]). Some have argued that these effects are inconsequential (see, for example, [10] [11] [12]), while some have argued the effect is real [13]. Buchert et al. [5] have disputed the general applicability of the Green and Wald [10] proof.

Yet homogeneity in the universe is still not well understood. For example, [14] have shown homogeneity in our universe over vastly larger volumes and scales than prior studies. Solutions to the general relativity equations in inhomogeneous scenarios are also still not well understood. For example, Giblin, Mertens and Starkman [15] have used numerical solution approaches to show a stronger impact from inhomogeneities, beyond what is expected from simpler mathematical solutions. Finally, Rácz et al. [16], have shown multiscale statistical solutions for a universe with many void regions that show accelerated expansion.

The debate continues. What remains clear is that inhomogeneities produce acceleration, and yet we know neither the magnitude of the inhomogeneities in the universe nor how to solve Einstein's equations of general relativity accurately in this complex "Swiss-cheese" universe.

Is there a way to maintain our concept of the homogeneous isotropic universe at very large scale which has served us so well and still generate an accelerated expansion effect from inhomogeneity beyond the Hubble sphere or even the observable universe?

This paper proposes an approach that preserves our isotropic homogeneous universe but surrounds it, at scale on the order of the observable universe, by another isotropic homogeneous section-perhaps best described as a thick shell universe versus the traditional, more locally inhomogeneous Swiss-cheese universe discussed above. 
This geometry violates the Copernican principle. However, the geometry maybe appropriate because repeated observations of the Cosmic Microwave Background (CMB) show an anisotropy, perhaps centered around the plane of the ecliptic [17] [18] [19].

In Section 2, we state the hypothesis. In Section 3, we develop the mathematical model for such a universe and in Section 4, we numerically solve the equations to explore how well the model can account for the observed acceleration. In Section 5, we discuss how such a universe could develop naturally as our assumed $\Lambda$ CDM model universe if we posit different initial conditions.

\section{Hypothesis}

The geometric hypothesis used thus far for the universe envisions an isotropic spherical expanding space. According to this hypothesis, the expanding universe is always converting its initial kinetic energy into potential energy. This naturally results in continuous deceleration of the universe throughout its history.

As we have seen above, one can add repulsive energy or modify the equations of gravitational theory to produce acceleration. But can the same result be achieved by altering the geometry and mass distribution at the beginning of the universe? What if our isotropic spherical expanding space universe was surrounded by a shell of different density, and this shell was beyond the observable universe? For a shell with decreasing mass, the modified equation for the expansion of the spherical space inside the shell would show an acceleration term due to the decreasing mass of the shell. Can this account for the observations of an accelerating universe?

\section{Theoretical Development}

\subsection{Standard Universe}

For the case of an isotropic spherical expanding universe it has been shown that the key aspects of the solution can be understood with purely Newtonian dynamics, as it generates almost the identical Friedmann equation [3]. In general relativity, the universe and space expand together; in the Newtonian treatment, we imagine a homogeneous sphere of matter expanding isotropically into existing empty Euclidian space. The sphere has an edge, a center of symmetry and a fixed mass.

Assume a sphere of mass $M_{T}$ and radius $r_{s}$ that expands with time. We consider the effect on a small mass at the very edge of the sphere. The gravitational force on the small mass is caused by the same constant mass $M_{T}$ enclosed at all times. Equating the gravitational force to mass times acceleration and solving for the velocity $v_{s}$ of the small mass, we obtain [20]:

$$
v_{s}^{2}=\frac{2 G M_{T}}{r_{s}}-k_{1},
$$

where $G$ is the gravitational constant and $k_{1}$ is a constant representing twice the total energy of a unit of mass. The rate of change of the radius $r_{s}$ of the sphere 
(our universe) is equal to $V_{S}$.

It is well known that Equation (1) has three solutions, depending on $k_{1}$ [20]: a closed universe that will expand and then contract for positive value of $k_{1}$; a zero-curvature universe that will expand into a "whimper" (parabolic expansion) for $k_{1}$ equal zero; and a more open universe that will also continue to expand (hyperbolic expansion) for a negative value of $k_{1}$. In all cases, expansion is decelerating right from the beginning as $r_{s}$ increases, driving $v_{s}$ lower and lower.

\subsection{Proposed Universe}

We now proceed to develop the solution for a different proposed universe. We will continue to use Newtonian mechanics, as we maintain our isotropic spherical geometry and Newtonian mechanics has been shown to lead to the same result as general relativity for a "spherical symmetric dust solution" [21].

Our proposed universe now consists of a similar homogeneous isotropic uniform sphere but with a shell of uniform density surrounding it, as depicted in Figure 1. We make no assumptions about the thickness of the shell versus the sphere's radius and we allow the structure to expand. We imagine a mass existing just outside the shell at $r_{s}$ to determine the behaviour of the outside radius, and we consider the expansion of the inner radius of the shell to see how the radius $r$ of our universe develops with time. In this universe, we allow $M_{1}$ and $M_{2}$ to be different but their sum, $M_{T}$, the total mass, to be constant.

The outer radius $r_{s}$ of the sphere is given by Equation (1), as the enclosed mass and spherical geometry are the same as before and the different distribution of mass does not affect the solution. Thus, the outside of this sphere decelerates with time, depending on $k_{1}$.

To understand the behaviour of the inner radius $r$, we proceed to calculate the gravitational force from the enclosed sphere of mass $M_{1}$ on a very thin section of the shell of mass $m$ at radius $r$ and equate it to the rate of change of its momentum. The sphere behaves as if all its mass, $M_{1}$, is at its center. Thus, the force on the section of the shell of mass $m$ is given by

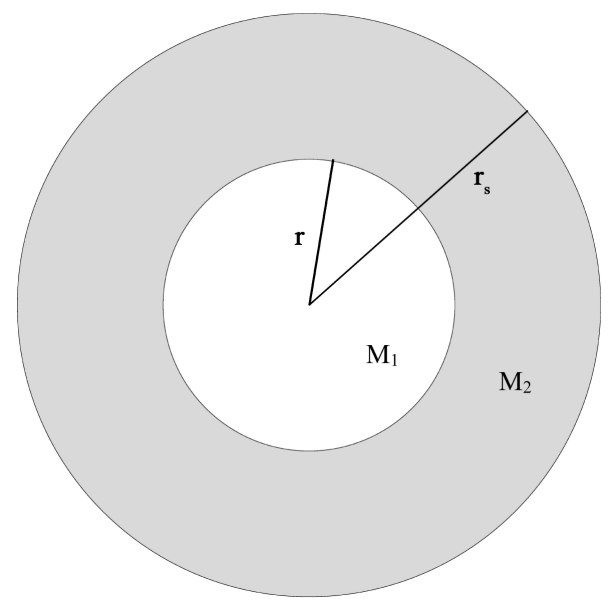

Figure 1. Proposed universe. 


$$
-\frac{G M_{1} m}{r^{2}}
$$

The rate of change of momentum of the thin section is

$$
\frac{\mathrm{d} m v}{\mathrm{~d} t}=v \frac{\mathrm{d} m}{\mathrm{~d} t}+m \frac{\mathrm{d} v}{\mathrm{~d} t}
$$

where $v$ is the rate of change of radius $r$. We assume that the mass of the thin section is proportional to $M_{2}$, an assumption that is more accurate if $M_{2}$ is changing slowly (Alternatively, one can assume that the density of the thin section is proportional to the density of the shell. The author obtained similar results with this assumption). Thus, Equation (3) becomes:

$$
\frac{\mathrm{d} m v}{\mathrm{~d} t}=v \alpha \frac{\mathrm{d} M_{2}}{\mathrm{~d} t}+\alpha M_{2} \frac{\mathrm{d} v}{\mathrm{~d} t}
$$

where $\alpha$ is a constant.

Combining Equations (2) and (4) we obtain:

$$
\frac{\mathrm{d}^{2} r}{\mathrm{~d} t^{2}}=-\frac{G M_{1}}{r^{2}}-\frac{1}{M_{2}} \frac{\mathrm{d} M_{2}}{\mathrm{~d} t} \frac{\mathrm{d} r}{\mathrm{~d} t}
$$

As the sphere expands, it gains mass:

$$
\mathrm{d} M_{1}=4 \pi r^{2} \rho_{2} \mathrm{~d} r
$$

where $\rho_{2}$ is the density of the shell. Thus, the change in $M_{1}$, which is equal to the negative of the change of $M_{2}$ (as the total mass remains constant), is given by

$$
\mathrm{d} M_{1}=-\mathrm{d} M_{2}=4 \pi r^{2} \rho_{2} \frac{\mathrm{d} r}{\mathrm{~d} t} \mathrm{~d} t
$$

We can calculate $\rho_{2}$ straightforwardly from $M_{2}, r_{s}$ and $r$ as follows:

$$
\rho_{2}=\frac{M_{2}}{(4 / 3) \pi\left(r_{s}^{3}-r^{3}\right)}
$$

We are left with the following set of equations to solve together:

- Equation (1)-providing $r_{s}$ as a function of time, with $M_{T}$ and $k_{1}$ being free variables to fit the observed data;

- Equation (5)-providing $r$ as a function of time, with $M_{1}, r$ and $v$ ( $r^{2}$ s rate of change) at some time being free variables to fit the data (in particular, we can obtain the rate of change of $r$ over $r$ at the current time from the Hubble constant); and

- Equation (7) - combined with Equation (8) - providing the increase of $M_{1}$ and decrease of $M_{2}$.

Thus, to run the equations we allow $M_{T}, k_{1}, r_{s}$ and $M_{1}$ to vary for the best fit (to the $\Lambda$ CDM universe with dark matter). We set the current rate of change in $r$ over $r$ to the current value of the Hubble constant.

It is clear from Equation (5) that for a decreasing $M_{2}$, there could well be periods where the acceleration term (the positive term on the right-hand side, when $M_{2}$ is decreasing) exceeds the deceleration term (the negative term on the right-hand side, due to gravity from $M_{1}$ ), producing the appearance of an accel- 
erating universe. It is also clear that the value of the acceleration term can be determined independently from the deceleration term by varying the input parameters above.

\section{Results}

We solve the set of equations numerically and plot the results in Figure 2. Figure 2 shows time along the $\mathrm{x}$ axis, with time zero being today. The $\mathrm{y}$ axis shows the scale factor of the universe, scaled to a value of 10 today. Displayed are curves for:

- A standard all-matter universe at critical mass (dash-dot line);

- The $\Lambda$ CDM universe (solid black line) with a Hubble constant of $67.8 \mathrm{~km} \mathrm{~s}^{-1}$ $\mathrm{Mpc}^{-1}$ and a matter component $\Omega_{\mathrm{M}}$ of 0.3089 and $\Omega_{\Lambda}$ of 0.6911 [4]; and

- The proposed universe $r$ (dotted line) and $r_{s}$ (dashed line).

We vary the parameters to provide the best fit possible of the proposed universe $(r)$ to the $\Lambda \mathrm{CDM}$ curve. We keep the proposed universe "flat" (although technically the acceleration term here has nothing to do with dark energy). The best fit is achieved with a current matter component $\Omega_{\mathrm{M}}$ of 0.38 (note that $\Omega_{\mathrm{M}}$ of 0.38 and $\Omega_{\Lambda}$ of 0.62 lie on the edge of the $95 \%$ confidence interval for CMB and supernova data [3]). This fit best fit is achieved for:

- $M_{T} 1.086$ times critical mass;

- $r_{s}$ of 1.803 the current scale factor value; and

- $k_{1}$ of -9.547 times the value of the mass term in Equation (1) at the current time, i.e., an open universe.

One can see that the fit is very good, and that the main effect is on the age of the universe, although in the proposed universe, the deceleration and acceleration components are a different function of radius than in the $\Lambda \mathrm{CDM}$ universe-in particular, the "cosmological" constant or acceleration parameter is not constant.

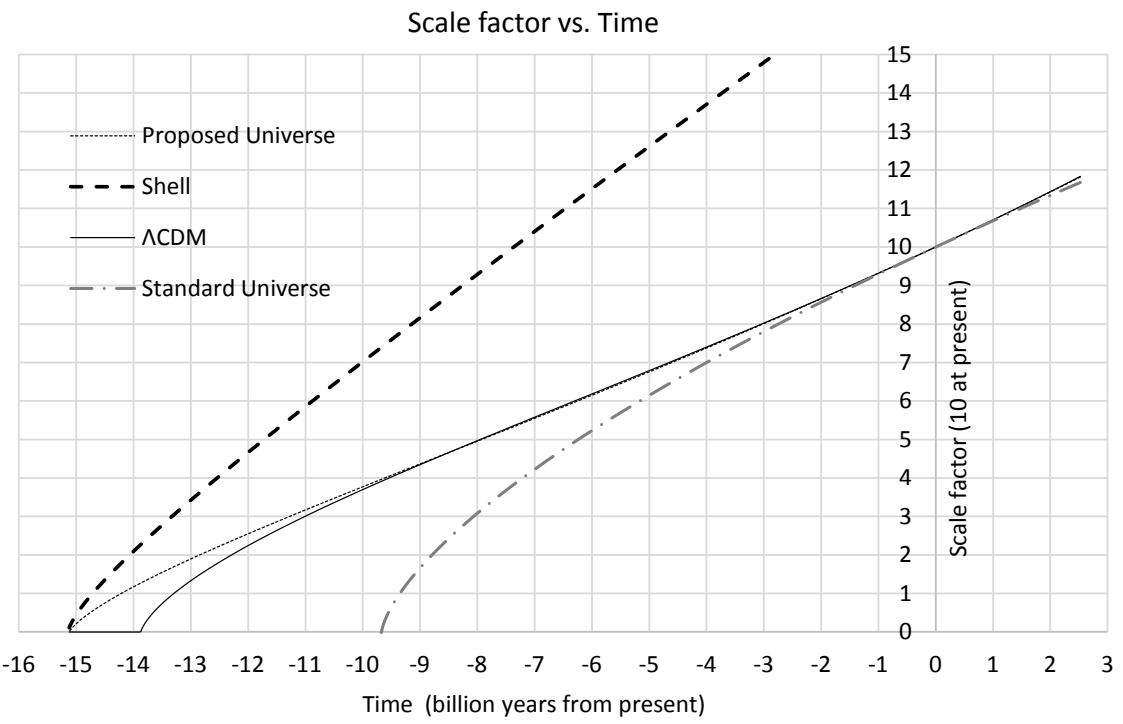

Figure 2. Scale factor vs. time for different universe models. 


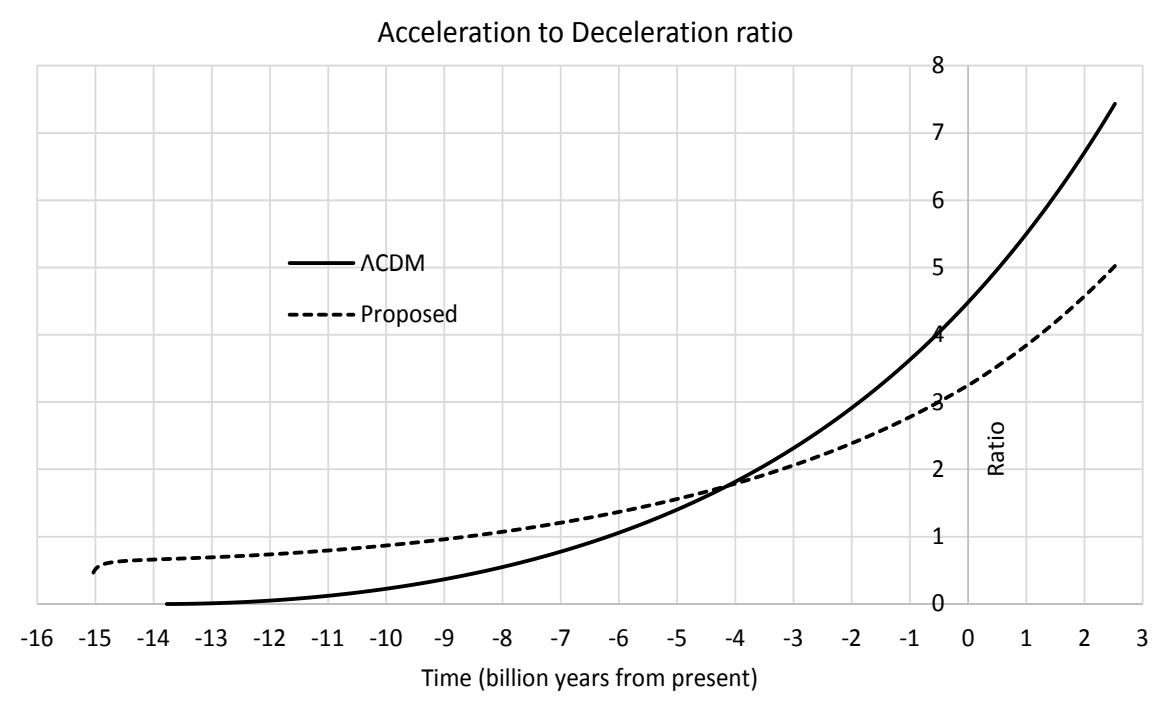

Figure 3. Ratio of acceleration to deceleration components.

A useful graph for seeing the difference in the nature of the acceleration and deceleration components between the proposed model and the $\Lambda \mathrm{CDM}$ model is shown in Figure 3, which displays the ratio of acceleration to deceleration components as a function of time for the proposed universe in Figure 2 and the $\Lambda \mathrm{CDM}$ universe. When the ratio is one, the universes flip from decelerating to accelerating. Clearly the fit in Figure 2 is achieved with different varying terms that integrate to almost the same result for times in the past 10 billion years. The proposed universe is gentler in terms of its switch from deceleration to acceleration.

\section{Mechanism}

The results described above have been achieved by fitting the data for the past several billion years and then running the model back in time. What emerges is the following picture:

- Initially, the mass of the universe is concentrated in the shell and the core is almost empty. However, the core quickly gains mass at the expense of the shell, and for the large majority of the universe's existence, the mass of the shell, $M_{2}$, slowly decreases, producing the effect required for acceleration. The masses of the two regions of the universe versus time are shown in Figure 4.

- Except at the very beginning of the universe, the density of the core is higher than the density of the shell, and the ratio of core density to shell density increases with time. At the current time, the core is 65 times denser than the shell.

- The ratio of $r$ to $r_{s}$ varies very slowly after the initial period, getting larger, as expected, in the past several billion years, as shown in Figure 5.

The acceleration effect is being produced by a universe like the one we currently imagine but surrounded outside its visible extent by (currently) a very low-density thick shell. 


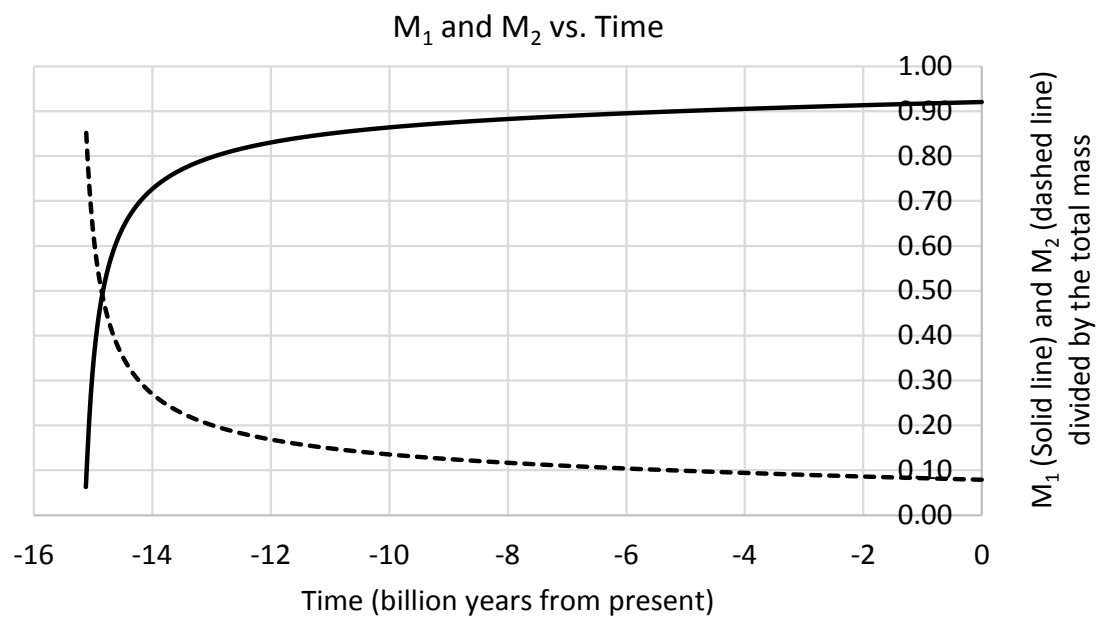

Figure 4. $M_{1}$ and $M_{2}$ as a function of time.

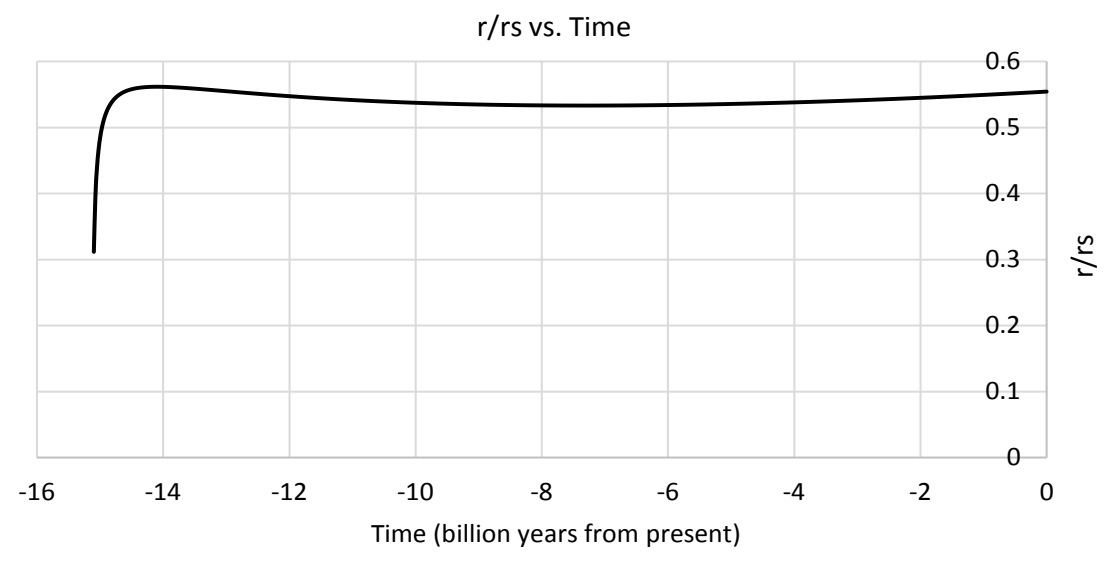

Figure 5. Ratio of $r$ to $r_{s}$ as a function of time.

\section{Conclusions}

It is demonstrated herein that by altering the geometry of the assumed expanding universe, one can achieve an initially decelerating universe and later in time an accelerating universe without inventing dark energy-simply by retaining visible and dark matter. Furthermore, the universe remains an expanding sphere that obeys Hubble's law. The process develops naturally from the initial conditions and explains how the universe has been accelerating for the past several billion years or so with no dark energy.

Three key pieces of work remain. The first is to perform a detailed fit to the experimental data, in particular the supernova data, by varying the parameters described herein. The second is to reproduce the theoretical result with the general relativity equations, and the last is to fully explore the initial conditions that make this approach work.

\section{Conflicts of Interest}

The authors declare no conflicts of interest regarding the publication of this paper. 


\section{References}

[1] Riess, A.G., Filippenko, A.V., Challis, P., et al. (1998) Observational Evidence from Supernovae for an Accelerating Universe and a Cosmological Constant. Astrophysical Journal, 116, 1009-1038.

[2] Conley, A., Guy, J., Sullivan, M., et al. (2011) Supernova Constraints and Systematic Uncertainties from the First Three Years of the Supernova Legacy Survey. The Astrophysical Journal, 192, Article ID: 1. https://doi.org/10.1088/0067-0049/192/1/1

[3] Ryden, B. (2017) Introduction to Cosmology. 2nd Edition, Chapters 4, 5 and 8, Cambridge University Press, Cambridge.

[4] Ade, P.A.R., Aghanim, N., et al. (2016) Planck 2015 Results XIII. Cosmological Parameters. Astronomy \& Astrophysics, 594, Article No. A13.

[5] Buchert, T., Coley, A.A., Kleinert, H., et al. (2016) Observational Challenges for the standard FLRW Model. International Journal of Modern Physics, D25, Article No. 1630007.

[6] Riess, A.G., Macri, L., Casertano, S., et al. (2011) A 3\% Solution: Determination of the Hubble Constant with the Hubble Space Telescope and Wide Field Camera 3. The Astrophysical Journal, 730, Article ID: 199.

[7] Joyce, A., Lombriser, L. and Schmidt, F. (2016) Dark Energy versus Modified Gravity. Annual Review of Nuclear and Particle Science, 66, 95-122. https://doi.org/10.1146/annurev-nucl-102115-044553

[8] Célérier, M.N. (2014) Effects of Inhomogeneities on the Expansion of the Universe: A Challenge to Dark Energy? Journal of Physics. Conference Series, 484, Article ID: 012005. https://doi.org/10.1088/1742-6596/484/1/012005

[9] Clarkson, C. (2012) Establishing Homogeneity of the Universe in the Shadow of Dark Energy. Comptes Rendus Physique, 13, 682-718. https://doi.org/10.1016/j.crhy.2012.04.005

[10] Green, S.R. and Wald, R.M. (2014) How Well Is Our Universe Described by an FLRW Model? Classical and Quantum Gravity, 31, Article ID: 234003.

[11] Green, S.R. and Wald, R.M. (2016) A Simple, Heuristic Derivation of Our "No Backreaction" Results. Classical and Quantum Gravity, 33, 12.

[12] Ishibashi, A. and Wald, R.M. (2006) Can the Acceleration of Our Universe Be Explained by the Effects of Inhomogeneities? Classical and Quantum Gravity, 23, 1. https://doi.org/10.1088/0264-9381/23/1/012

[13] Chuang, C.-H., Gu, J.-A. and Hwang, W.-Y. (2008) Inhomogeneity-Induced Cosmic Acceleration in a Dust Universe. Classical and Quantum Gravity, 25, 17. https://doi.org/10.1088/0264-9381/25/17/175001

[14] Zibin, J.P. and Moss, A. (2014) Nowhere to Hide: Closing in on Cosmological Homogeneity. arXiv:1409.3831v1.

[15] Giblin, J.T., Mertens, J.B. and Starkman, G.D. (2016) Departures from the Friedmann-Lemaitre-Robertston-Walker Cosmological Model in an Inhomogeneous Universe: A Numerical Examination. Physical Review Letters, 116, Article ID: 251301. https://doi.org/10.1103/PhysRevLett.116.251301

[16] Rácz, G., Dobos, L., Beck, R., et al. (2017) Concordance Cosmology without Dark Energy. MNRAS Letters, 469, 1.

[17] Chang, Z., Rath, P.K., et al. (2018) Anisotropic Power Spectrum and the Observed Low-L Power in PLANCK CMB Data. Astronomy \& Astrophysics, 18, 3.

[18] Ade, P.A.R., Aghanim, N., et al. (2016) Planck 2015 Results XVI. Isotropy and Sta- 
tistics of the CMB. Astronomy \& Astrophysics, 594, A16.

[19] Challinor, A. (2013) CMB Anisotropy Science: A Review. Proceedings of the IAU, 8 , 42-52.

[20] Harrison, E. (2003) Cosmology-The Science of the Universe. 2nd Edition, Chapter 16, Cambridge University Press, Cambridge.

[21] Szekeres, P. and Lun, A. (1999) What Is a Shell-Crossing Singularity? Journal of the Australian Mathematical Society, 41, 167. 